\title{
QUÍMICA ORGÁNICA PARA ESTUDIANTES DE INGENIERÍA DE MINAS E INGENIERÍA METALÚRGICA
}

\author{
MAURELIA GÓMEz, R. \\ Departamento de Ciencias Naturales, Facultad de Ingeniería, Universidad de Atacama, Casilla 240, \\ Copiapó (Chile).
}

\section{SUMMARY}

Organic Chemistry is usually related to agricultural, pharmaceutical, alimentary, petroleum and derivate industries among others. However, the use of organic substances in other different industrial fields, like mining and metallurgy has increased and it is hoped that this increment will go on in the future. In this paper, a brief review of this kind of substances was realized. Besides, an Organic Chemistry Program for Mining and Metallurgy Engineering students is proposed.

\section{INTRODUCCIÓN}

La incorporación creciente en la segunda mitad del siglo de poderosas técnicas instrumentales para dilucidar o confirmar estructuras, tales como la espectroscopía y la cristalografía de rayos $\mathrm{X}$ y el desarrollo de nuevos procedimientos preparativos, han imprimido un fuerte impuiso a la síntesis orgánica (Cohendet et al. 1989). A Io anterior debe agregarse el almacenamiento y manejo de la información a través de la informática, la robotización de las operaciones y la «funcionalización" de los métodos sintéticos. Esto último se refiere a considerar en ellos la función que deberán cumplir los productos, sus vías de eliminación, sus condiciones de empleo y eventualmente la valorización de los subproductos, concepto que ha trasladado la atención desde la química deI producto a la química de la función. Estos avances han posibilitado el desarrollo de la síntesis orgánica también desde el punto de vista industrial (Ourisson 1990) y han facilitado la obtención de una variada gama de compuestos cuyas aplicaciones alcanzan todas las ramas de la industria, siendo el sector minero-metalúrgico un ejemplo relevante. Ya en 1967 se estimaba que solamente las ventas de polímeros orgánicos a la industria minera norteamericana excedían los 4 miliones de dólares anuales y la proyección era de 40 millones al cabo de 10 años(McLean 1968).

Adicionalmente, debe señalarse que las actividades minero-metalúrgicas tienen la tendencia a hacerse «más químicas» debido a la evolución tecnológica de los procesos y a la disminución de las leyes de los yacimientos. Algunos especialistas han señalado la necesidad de expertos en termodinámica, cinética de procesos y equilibrios químicos entre los profesionales del sector para hacer frente a esta realidad (Sutulov 1978). La química orgánica se aproxima cada vez con más énfasis a estas prioridades y debe pensarse en incorporar algunos de sus elementos en los currículos de las carreras que involucran a este tipo de profesionales.

La introducción de elementos de química orgánica en los currículos de ingentería de minas e ingeniería metalúrgica, permitiría :

a) Facilitar a los estudiantes la comprensión de los procesos u operaciones minero-extractivas basados, o en los que participan, compuestos orgánicos.

b) Promover la interacción de ingenieros de minas y metalúrgicos con profesionales químicos en estudios interdiscipfinarios.

c) Reunir, en un cuerpo estructurado y coherente, contenidos actualmente dispersos en asignaturas de especialidad dentro del currículo.

d) Familiarizar a estudiantes de estas especialidades con materiales que tienden a reemplazar los metales en algunas aplicaciones. 


\section{DETERMINACIÓN DE CONTENIDOS}

En el presente trabajo se realiza una revisión de las etapas de obtención de minerales (ingeniería de minas) y su posterior procesamiento hasta la elaboración de piezas metálicas (ingeniería metalúrgica) (Peele 1927, Young 1955 y Schulz 1978). En esta secuencia se identifican Ios compuestos orgánicos utilizados y se especifica la función que cumplen en los procesos en que están insertos. En esta revisión se han considerado aplicaciones más bien específicas y no comunes a cualquier tipo de industria, como combustibles,lubricantes, etc. Esta información se resume en las tablas I y II (Kirk y Othmer 1962, McLean 1968, Karger et al. 1973, Catálogo Hoesch 1980, Jarufe 1986, Arriaza 1986, Catálogo Allied Colloids 1986).

La función orgánica a que pertenecen las sustancias identificadas, las propicdades físicas y químicas que sustentan su rol en los procesos respectivos y los aspectos estructurales que determinan dichas propiedades han servido de base para diseñar un Programa de Química Orgánica que en términos generales pueda satisfacer los requerimientos curriculares antes mencionados.

En esta proposición se ha tratado de observar una apropiada dosificación de contenidos con el objeto de que su eventual implementación,ya sea en carácter de asignatura obligatoria o electiva, no signifique un recargo de estos currículos, de suyo extensos y, más aún, de los cuales se piensa que deberán acentuar progresivamente su componente en otras áreas, como gestión y negocios (Whiting y Duncan 1988).

\section{ESTRUCTURA DEL PROGRAMA PRO- PUESTO}

\section{Introducción}

Naturaleza de la química orgánica. Estructura molecular y propiedades de las sustancias químicas: Representación de compuestos orgánicos, fómulas estructurales. Funciones orgánicas y grupos funcionales. Series homólogas.

\section{Hidrocarburos}

Nomenclatura. Características estructurales: Geometría molecular, isomería. Propiedades físicas. Fuente natural y obtención. Combustión y combustibles. Hidrocarburos como materias primas.

\section{Compuestos orgánicos oxigenados}

Alcoholes, fenoles, éteres, aldehídos y cetonas: Nomenclatura. Obtención industrial. Rasgos estructurales. Propiedades físicas y químicas. Importancia industrial. Compuestos 1,3 dicarbonílicos: equilibrio ceto-enólico, propiedades quelantes.

\section{Ácidos carboxílicos y derivados}

Ácidos y sales carboxílicas, cloruros đe ácidos, anhídridos, ésteres y amidas. Obtención. Aspectos estructurales. Propiedades físicas y químicas. Importancia industrial.

\section{Compuestos orgánicos nitrogenados}

Aminas, sales de amonio cuaternarias y nitrilos: Obtención. Aspectos estructurales. Propiedades físicas y químicas. Importancia industrial.

\section{Compuestos organosulfurados, organofosforados y polifuncionales}

Nomenclatura de compuestos orgánicos polifuncionales. Ésteres de los ácidos carbónico, nítrico, carbámico, sulfúrico, sulfónico y fosfónico y sus respectivos derivados sulfurados : Nomenclatura, propiedades físicas y químicas.

\section{Polímeros y polimerización}

Conceptos preliminares. Clasificación de polímeros. Propiedades físicas y estructura. Procesos de polimerización. Polímeros naturales.

\section{Aplicaciones de los productos orgánicos a la minería}

\section{Introducción}

Extractantes líquido-líquido y sólido-líquiđo de iones metálicos en solución. Explosivos. Reactivos de flotar ción. Floculantes y dispersantes.

En el primer capítulo se dan los elementos introductorios a la química orgánica, su naturaleza, campo de acción y vinculación con otras ciencias. Se clasifican los compuestos orgánicos y se indican formas de representarlos.

Entre los capítulos 2 y 7 , se describen Ias funciones orgánicas más relevantes, su nomenclatura IUPAC y trivial, propiedades físicas y químicas, la forma de obtenerlas desde fuentes naturales o sintéticas; como asimismo los compuestos de mayor importancia industrial y sus aplicaciones de mayor interés.

Dada la diversidad de aplicaciones de polímeros a la industria minera y metalúrgica, estos compuestos se tratan en un capítulo aparte (cap. vir), se clasifican, se describen sus propiedades y las diferentes reacciones de polimerización.

La orientación que se pretende dar al programa se fundamenta en el capítulo viI, donde se discute la participación de compuestos orgánicos en determinadas operaciones importantes de esta rama de la industria. Sin perjuicio de lo anterior, cuando se estudian las distintas funciones orgánicas en los capítulos 2 al 7 , se hace mención a 
Tabla I

Sustancias orgánicas utilizadas en ingeniería de minas.

\begin{tabular}{|c|c|c|c|}
\hline $\begin{array}{l}\text { Etapa de la obtención } \\
\text { de minerales }\end{array}$ & Operaciones involucradas & Función & Sustancias orgánicas \\
\hline 1. Prospeccion & - & - & - \\
\hline 2. Reconocimiento & Sondaje & $\begin{array}{l}\text { Aditivos para lodos: viscosi- } \\
\text { ficantes, emulsificantes }\end{array}$ & $\begin{array}{l}\text { Cortes de petróleo, aimidón } \\
\text { carboximetilceluiosa, } \\
\text { sulfonatos de lignina }\end{array}$ \\
\hline 3. Planificación & - & - & - \\
\hline \multirow[t]{11}{*}{$\begin{array}{l}\text { 4. Preparacione } \\
\text { infraestructura }\end{array}$} & \multirow[t]{4}{*}{$\begin{array}{l}\text { Construcción superficial } \\
\text { o subterránea }\end{array}$} & $\begin{array}{l}\text { Acondicionamiento y con- } \\
\text { solidación de suelos } \\
\text { arenosos }\end{array}$ & $\begin{array}{l}\text { Resinas epóxidas, copolímeros } \\
\text { de acrilamida }\end{array}$ \\
\hline & & $\begin{array}{l}\text { Rellenos químicos para } \\
\text { pernos de anclaje }\end{array}$ & $\begin{array}{l}\text { Resinas de poliéster, polia- } \\
\text { crilamidas }\end{array}$ \\
\hline & & $\begin{array}{l}\text { Rellenos de formaciones } \\
\text { rocosas fracturadas }\end{array}$ & Resinas epóxidas, poliésteres \\
\hline & & $\begin{array}{l}\text { Cubiertas protectotas } \\
\text { contra erosión y generación } \\
\text { de polvos en techos y } \\
\text { túneles }\end{array}$ & $\begin{array}{l}\text { Poliesteres, poliuretanos, } \\
\text { resinas epóxidas } \\
\text { (emulsiones) }\end{array}$ \\
\hline & \multirow[t]{3}{*}{ Labores mineras } & $\begin{array}{l}\text { Sellantes de fisuras y } \\
\text { filtraciones de agua }\end{array}$ & $\begin{array}{l}\text { Resinas epóxidas, poliureta- } \\
\text { nos clorosulfonado }\end{array}$ \\
\hline & & $\begin{array}{l}\text { Cañerías antiácidos para } \\
\text { drenajes de agua de mina }\end{array}$ & $\begin{array}{l}\text { P.V.C., poliuretanos, polie- } \\
\text { tileno clorosulfonado }\end{array}$ \\
\hline & & $\begin{array}{l}\text { Recubrimiento de cañerías } \\
\text { contra corrosión y abrasión }\end{array}$ & Resinas epóxidas, PVC \\
\hline & \multirow[t]{4}{*}{$\begin{array}{l}\text { Instalaciones de } \\
\text { equipos }\end{array}$} & $\begin{array}{l}\text { Protección antiimpacto } \\
\text { de equipo pesado }\end{array}$ & Poliésteres \\
\hline & & Cañerías de ventilación & $\begin{array}{l}\text { Polietileno, resinas de } \\
\text { poliéster }\end{array}$ \\
\hline & & $\begin{array}{l}\text { Sellantes y aislantes } \\
\text { térmicos de ventilación }\end{array}$ & Poliuretanos, policroropreno \\
\hline & & Retardantes de llama & Poliuretanos \\
\hline \multirow[t]{4}{*}{ 5. Explotación } & Perforación (petrolífera) & $\begin{array}{l}\text { Emulsificantes, viscosifi- } \\
\text { cantes y anticontaminan- } \\
\text { tes de lodos de perforación }\end{array}$ & $\begin{array}{l}\text { Sales sódicas de terpineoles } \\
\text { y carboximetilcelulosa, } \\
\text { ałmidón, fenoles, polioxie- } \\
\text { tileno, sulfonatos de lignina }\end{array}$ \\
\hline & Tronadura & Explosivos & $\begin{array}{l}\text { Derivados nitrados de polio- } \\
\text { les, fenoles, hidrocarburos } \\
\text { aromáticos y celulosá }\end{array}$ \\
\hline & Carguío & - & - \\
\hline & Transporte & $\begin{array}{l}\text { Aglomeraciones de finos } \\
\text { para prevenir pérdidas } \\
\text { por viento y lluvia }\end{array}$ & $\begin{array}{l}\text { Copolímeros de: estireno, } \\
\text { butadieno, acrilato de } \\
\text { etilo }\end{array}$ \\
\hline
\end{tabular}


Tabla II

Sustancias orgánicas utilizadas en ingeniería metalúrgica.

\begin{tabular}{|c|c|c|c|}
\hline Área de metalurgia & $\begin{array}{l}\text { Operación o proceso } \\
\text { unitario }\end{array}$ & Función & Sustancias orgánicas \\
\hline $\begin{array}{l}\text { 1. Metalurgia } \\
\text { extractiva }\end{array}$ & $\begin{array}{l}\text { Chancado, molienda, } \\
\text { cribado }\end{array}$ & $\begin{array}{l}\text { Aglomerantes de finos } \\
\text { para contrarrestar formación } \\
\text { de polvos en conminución y } \\
\text { pérdidas en almacenamiento }\end{array}$ & $\begin{array}{l}\text { Copolimeros de estireno, buta } \\
\text { dieno, acrilato de etilo }\end{array}$ \\
\hline \multirow[t]{10}{*}{$\begin{array}{l}\text { 1.1. Preparación de } \\
\text { de minerales }\end{array}$} & & $\begin{array}{l}\text { Rellenos y recubrimientos } \\
\text { antímpacto y revestimientos } \\
\text { antiabrasión }\end{array}$ & $\begin{array}{l}\text { Resinas de poliéster, poliu- } \\
\text { retanos, PVC }\end{array}$ \\
\hline & Flotación & Colectores & $\begin{array}{l}\text { Xantatos y ditiofosfatos alca- } \\
\text { linos, tionocarbamatos, tio- } \\
\text { carbamatos, tiocarbanilida, } \\
\text { xantoformiatos, sulfonatos } \\
\text { alcalinos, acidos grasos, } \\
\text { aminas, sales de amonio cua- } \\
\text { ternarias }\end{array}$ \\
\hline & & Espumantes & $\begin{array}{l}\text { Alcoholes naturales y sinté- } \\
\text { ticos, fenoles, parafinas alcoxi } \\
\text { sustituidas, cetonas }\end{array}$ \\
\hline & & $\begin{array}{l}\text { Modificadores, recubridores, } \\
\text { depresantes }\end{array}$ & $\begin{array}{l}\text { Poliacrilamida, dextrina, } \\
\text { almidón, carboximetil- } \\
\text { celulosa }\end{array}$ \\
\hline & $\begin{array}{l}\text { Filtrado, sedimentación y } \\
\text { transporte hidraúlico }\end{array}$ & Floculantes & $\begin{array}{l}\text { Polímeros de la acrilamida y } \\
\mathrm{N}, \mathrm{N} \text {-metilenbisacrilamida }\end{array}$ \\
\hline & & $\begin{array}{l}\text { Dispersantes, «antiqueque» } \\
\text { y anticongelantes }\end{array}$ & $\begin{array}{l}\text { Aminas, policrilamidas de } \\
\text { bajo peso molecular }\end{array}$ \\
\hline & & $\begin{array}{l}\text { Cañerías antiformación } \\
\text { de depósitos }\end{array}$ & PVC \\
\hline & Lixiviación & Lixiviantes & Tiourea (para Au y Ag) \\
\hline & & $\begin{array}{l}\text { Telas impermeables } \\
\text { para evitar percolación } \\
\text { de soluciones } \\
\text { tóxicas }\end{array}$ & Poliuretanol \\
\hline & & $\begin{array}{l}\text { Cañerías, fittings y } \\
\text { revestimientos resistentes } \\
\text { a la abrasión, corrosión } \\
\text { y ácidos }\end{array}$ & $\begin{array}{l}\text { Resinas epóxidas, polietileno } \\
\text { y polipropileno de alta } \\
\text { densidad, PVC de alto } \\
\text { impacto, poliuretano, } \\
\text { polietileno clorosulfurado. }\end{array}$ \\
\hline \multirow[t]{2}{*}{ 1.2. Procesos unitarios } & $\begin{array}{l}\text { Electrorecuperacion } \\
\text { y electrorefinación }\end{array}$ & $\begin{array}{l}\text { Reguladores (inhibidores) } \\
\text { de electrodepositación }\end{array}$ & $\begin{array}{l}\text { Polímeros de acrilamida, } \\
\text { tiourea, derivados de la } \\
\text { lignina, caseina, sulfonatos } \\
\text { de sodio, aminas aromáticas }\end{array}$ \\
\hline & Proceso hidrometalúrgico & $\begin{array}{l}\text { Solventes y extractantes de } \\
\text { especies metálicas desde } \\
\text { solución acuosa }\end{array}$ & $\begin{array}{l}\text { Cortes de petróleo, hidroxila- } \\
\text { minas, hidroxioximas, hidro- } \\
\text { xiquinolinas, aminas, sales de } \\
\text { amonio cuaternatias, ésteres } \\
\text { y óxidos del ácido fosfórico, } \\
\text { ácidos carbolxilicos, ésteres, } \\
\text { cetonas }\end{array}$ \\
\hline
\end{tabular}




\begin{tabular}{|c|c|c|c|}
\hline Área de metalurgia & $\begin{array}{l}\text { Operación o proceso } \\
\text { unitario }\end{array}$ & Función & Sustancias orgánicas \\
\hline & \multirow{6}{*}{ Fusión y colada } & $\begin{array}{l}\text { Resinas de intercambio } \\
\text { iónico } \\
\text { Cañerias y revestimiento } \\
\text { para soluciones corrosivas } \\
\text { y líquidos orgánicos }\end{array}$ & $\begin{array}{l}\text { Resinas de copolímeros } \\
\text { estireno-divinilbenceno } \\
\text { sulfonadas y aminadas } \\
\text { PVC alto impacto, poliu- } \\
\text { retano }\end{array}$ \\
\hline $\begin{array}{l}\text { 2. Metalurgia } \\
\text { adaptiva }\end{array}$ & & $\begin{array}{l}\text { Aglomerantes de arenas } \\
\text { para moldes y almas }\end{array}$ & $\begin{array}{l}\text { Resinas amínicas, fenolicas, } \\
\text { alquídicas, arńlicas feno- } \\
\text { formaldehido, urea-formal- } \\
\text { dehido y polímeros furánicos }\end{array}$ \\
\hline \multirow[t]{4}{*}{$\begin{array}{l}\text { 2.1. Procesamiento } \\
\text { de metales }\end{array}$} & & Pelletizadores & $\begin{array}{l}\text { Copolímeros de acrilamida } \\
\text { y derivados de celulosa }\end{array}$ \\
\hline & & $\begin{array}{l}\text { Construcción de matrices } \\
\text { para moldes }\end{array}$ & $\begin{array}{l}\text { Resinas epóxidas, pliacri- } \\
\text { lamidas, poliuretanos, polí- } \\
\text { meros vinílicos, resinas de } \\
\text { poliéster }\end{array}$ \\
\hline & & $\begin{array}{l}\text { Rellenos de moldeo en } \\
\text { Full Mold Process }\end{array}$ & Espumas de poliestireno \\
\hline & & $\begin{array}{l}\text { Antiprecipitantes para } \\
\text { agua en camisas de } \\
\text { refrigeración de hornos }\end{array}$ & Polímeros de acrilamida \\
\hline $\begin{array}{l}\text { 2.2. Metalurgia de } \\
\text { aplicación }\end{array}$ & - & - & - \\
\hline
\end{tabular}

aplicaciones específicas; por ejemplo, cuando se tratan los alcoholes y fenoles, se describen algunos que se emplean como espumantes y otros que como derivados nitrados se utilizan como explosivos, etc.

Atendiendo a que tanto los compuestos químicos estudiados como también las operaciones industriales en las que se insertan son frecuentemente vinculados a problemas ambientales, este aspecto es incorporado en el desarrollo del curso. Aśí, ya en et capítulo introductorio se destaca que, si bien algunos compuestos orgánicos como por ejemplo los halogenuros de alquilo pueden contribuir al desarrollo tecnológico y al confort de la humanidad en aplicaciones como refrigerantes opropelentes de lacas, pinturas, aromatizantes, etc., del mismo modo, utilizados en gran escala, pueden desencadenar proble. mas ambientales severos desde un punto de vista global, como, en este caso, la alteración del ciclo natural del ozono atmosférico.

Determinados temas son propicios para incorporar Ia dimensión ambiental. Tal es el caso del capítulo de hidrocarburos, donde en forma natural emergen los pro- blemas de desaparición de fuentes naturales no renovables, necesidades de fuentes energéticas alternativas a los combustibles fósiles, agudización del efecto invernadero por aumento de $\mathrm{CO}$, generado en las combustiones, entre otros. En el capítulo de polímeros se menciona la resistencia a la biodegradabilidad de los plásticos. El mismo aspecto se destaca en la discusión de compuestos organosulfurados y organofosforados tensoactivos utilizados como detergentes sintéticos, incluyendo, en este útimo caso, como consecuencia, la eutroficación de recursos hídricos.

Durante todo el desarrollo del programa, al describir las propiedades de los compuestos orgánicos, cuando es del caso, se pone énfasis en su actividad biológica, para inferir precauciones de manejo por parte de eventuales usuarios, tanto en su manipulación, como en la disposición de sus residuos.

Finalmente, así como se describen riesgos y problemas ambientales generados por sustancias orgánicas especialmente en el capítulo vm, del mismo modo se menciona el uso de algunos de ellos en acciones remediales 
de dichos problemas. Por ejemplo el empleo de aerosoles poliméricos para prevenir generación de polvo en ambientes subterráneos y otras faenas, resinas quelantes o intercambiadoras que pueden disminuir descargas metalogénicas de soluciones residuales emitidas al ambiente, polímeros hidrosolubles floculantes que clarifican aguas industriales, etc.

Este curso ha sido diseñado para un semestre de duración (18 semanas lectivas) con 4 a 5 horas semanales. Como prerequisito exige Química General y es deseable que Ios alumnos se hayan interiorizado de sus respectivas

\section{REFERENCIAS BIBLIOGRÁFICAS}

ALLIED COLLOIDS, LTD., 1986. Catálogo «Allied Colloids Health, and Safety Information». (Yorkshire).

ARRIAZA, M., 1986. Estudio de Inhibidores en Electrorefinación de Cobre. Trabajo de titulación para ingeniero de ejecución (Universidad de Atacama: Copiapó).

COHENDET, P., HERAUD, J.A. y LEDOUX, M.J., 1989. Quelle Chimie Pour L'An 2000?, La Recherche, Vol. 20,pp 1254.

HOECHST, 1980. Catálogo Hoechst: Flotations reagenzien: Frankfurt.

JARUFE, K., 1987. Extracción por Solventes: Nueva técnica para recuperar metales, Minería Chilena, 77, p. 33.

KARGER, K., SNYDER, L. y HORVALTH, C., 1973. An Introduction to Separation Science. (John Wiley \& Sons: Nueva York).

KIRK, R., OTHMER, D., 196I. Enciclopedia de Tecnología Quimica, Vol. 11, pp. 881. (UTEHA: México). especialidades, ya que esto facilita el logro de los objeiivos que se propone su formulación.

\section{AGRADECIMIENTOS}

El autor desea expresar su agradecimiento a los profesores Ángel Astorga y Enrique Santibáñez de los Departamentos de Ingenierfa Metalúrgica e Ingeniería de Minas de la Universidad de Atacama respectivamente, por sus valiosos aportes y sugerencias.
MCLEAN, D.C., 1968. Enciclopedia of Polymer Science and Technology, Vol. 2, pp. 798-812. (John Wiley \& Sons: Nueva York).

OURISSON, P. G., 1990. Sans La Chemie, Rien Ne Va, La Recherche, Vol. 21, pp. 1159.

PEELE, R., 1927. Mining Engineers Handbook. (John Witey \& Sons Inc.: Londres).

SCHULZ, B., 1978. Introducción a la Metalurgia. (Publicación Interna. Departamento de Metalurgia Univerșidad Técnica del Estado. Santiago. Chile).

SUTULOV, A., 1978. Editorial: «Química y Minería», Revista Chilena Educación Química, Vol. 3 (4), pp. 202.

WHITING, J., DUNCAN N., 1988. Educating Mining Engineers for the 21 st Century, CIM Bulletin, Vol. 81 (914), pp. 41 .

YOUNG, G.J., 1955. Elementos de Minería. (Gustavo Gili: Barcelona). 